\title{
Developmental Programming: Priming Disease Susceptibility for Subsequent Generations
}

\author{
L. C. Messer • J. Boone-Heinonen • L. Mponwane • \\ L. Wallack • K. L. Thornburg
}

Published online: 8 January 2015

(C) Springer International Publishing AG 2015

\begin{abstract}
Racial and/or ethnic minorities carry the highest burden of many adverse health outcomes intergenerationally. We propose a paradigm in which developmental programming exacerbates the effects of racial patterning of adverse environmental conditions, thereby contributing to health disparity persistence. Evidence that developmental programming induces a heightened response to adverse exposures ("second hits") encountered later in life is considered. We evaluated the evidence for the second hit phenomenon reported in animal and human studies from three domains (air, stress, nutrition). Original research including a gestational exposure and a childhood or adulthood second hit exposure was reviewed. Evidence from animal studies suggest that prenatal exposure to air pollutants is associated with an exaggerated reaction to postnatal air pollution exposure, which results in worse health outcomes. It also indicates offspring exposed to prenatal $m a-$ ternal stress produce an exaggerated response to subsequent stressors, including anxiety and hyper-responsiveness of the hypothalamic-pituitary-adrenal axis. Similarly, prenatal and postnatal Western-style diets induce synergistic effects on weight gain, metabolic dysfunction, and atherosclerotic risk. Cross-domain second hits (e.g., gestational air pollution followed by childhood stressor) were also considered. Suboptimal gestational environments induce exaggerated offspring
\end{abstract}

This article is part of the Topical Collection on Social Epidemiology

L. C. Messer $(\bowtie) \cdot$ L. Mponwane $\cdot$ L. Wallack

School of Community Health, College of Urban and Public Affairs, Portland State University, Portland, OR, USA

e-mail: lynne.messer@pdx.edu

J. Boone-Heinonen

Department of Public Health and Preventive Medicine, Oregon

Health \& Science University, Portland, OR, USA

K. L. Thornburg

Moore Institute, Oregon Health \& Science University, Portland, OR, USA responses to subsequent environmental and social exposures. These developmental programming effects may result in enhanced sensitivity of ongoing, racially patterned, adverse exposures in race/ethnic minorities, thereby exacerbating health disparities from one generation to the next. Empirical assessment of the hypothesized role of priming processes in the propagation of health disparities is needed. Future social epidemiology research must explicitly consider synergistic relationships among social environmental conditions to which gestating females are exposed and offspring exposures when assessing causes for persistent health disparities.

Keywords Developmental programming $\cdot$ Health disparities $\cdot$ Social epidemiology $\cdot$ Priming $\cdot$ Air pollutants · Psychosocial stress · Diet

\section{Introduction}

Not long ago, scientists conceived of infants as unadulterated by historic behaviors or environmental insults but now understand that babies are record keepers of maternal and paternal exposures to environmental conditions [1-3]. We propose that this record keeping at least partially occurs through a biological process known as developmental programming whereby racial disparities in health are not only propagated [4-7] but, when combined with persistent environmental exposures, are magnified from one generation to the next.

Developmental programming, or developmental origins of health and disease, refers to the changes in fetal structure and physiology that result from adverse early life exposures that increase susceptibility to poor health outcomes later in life [8]. Programmed characteristics are believed to be largely stable 
after the postnatal period and have been associated with increased risk of obesity, cancer, and heart disease, among others [9]. The maternal stressors that serve as exposures associated with developmental programming include environmental pollutants $[10,11]$, social stress $[12,13]$, and dietary restriction or high-calorie malnutrition [14-17].

\section{Conceptual Framework}

Decades of social epidemiologic research demonstrate that race and ethnic minorities face greater exposure to adverse social and environmental health determinants, resulting in health disparities. These disparities are persistent across generations and have been studied in numerous domains (e.g., environmental injustice, neighborhood deprivation, lower quality educational opportunities), yet the mechanisms underlying their proliferation remain unknown. We propose that developmental programming is a critical, previously overlooked factor in the propagation of health disparities from one generation to the next.

Figure 1 illustrates a paradigm of developmental programming on health, according to ongoing environmental exposures, across three generations. Health status in the first $\left(\mathrm{F}_{0}\right)$ generation is determined by a woman's lifetime accumulation of adverse exposures; $\mathrm{D}_{0}$ denotes the health status for women in generation $\mathrm{F}_{0}$ who experience adverse exposures, and $\mathrm{A}_{0}$ denotes the health status of a woman whose exposure profile is healthier. It is well known that adverse exposures are more common at greater levels of social and economic disadvantage. Therefore, we characterize trajectories stemming from exposure likelihood in the baseline generation as representing disadvantaged (D) and advantaged (A) trajectories.

If no further harmful exposures were incurred in subsequent generations, health trajectories could be expected to remain somewhat constant, traveling along the solid lines in the figure, resulting in positions $A_{10}, A_{20}, D_{10}$, and $D_{20}$. Because disadvantage accumulates over time, one would expect the health of disadvantaged women to decline over their lifetimes. Furthermore, the weathering hypothesis, originally conceived to explain the premature worsening of health by African-American women as a consequence of cumulative social disadvantage, can be applied here as the acceleration of disease progression within a woman's life course [18]. $\alpha_{\mathrm{g}}$ denotes the difference in health between the advantaged and disadvantaged trajectories in generation $\mathrm{g}$.

$\beta$ represents the decrement in health caused by an ongoing adverse exposure; both advantaged and disadvantaged women move to a position of worse health when so exposed. The $F_{1}$ generation communicates this health status to any developing offspring in generation $F_{2}$, who are then born from the combined baseline maternal risk $\left(\mathrm{A}_{10}\right.$ or $\left.\mathrm{D}_{10}\right)$ supplemented by effects of any adverse exposures accumulating prior to or during pregnancy $\left(\beta_{1}\right.$ or $\left.\beta_{1}^{*}\right)$. The scenario in which adverse exposures persist from one generation to the next produces an increasing health gap over time ( $\alpha_{1}$, represented by the difference between $A_{11}$ and $D_{11}$; and $\alpha_{2}$, the difference between $A_{21}$, and $D_{21}$ ). Such persistent exposures observed in the social epidemiology literature include the intergenerational
Fig. 1 Theoretical role of developmental programming in propagation and magnification of health disparities over three generations. $A_{g x}$ and $D_{g x}$ denote health status $Y_{g x}$ for generation $\mathrm{g}$ and exposure status x. $\beta_{g}$ and $\beta_{g *}$ denote the difference in health on $\mathrm{Y}_{\mathrm{gx}}$ between advantaged and disadvantaged trajectories. $\alpha_{g}$ denotes the magnitude of disparity in any in generation $g$

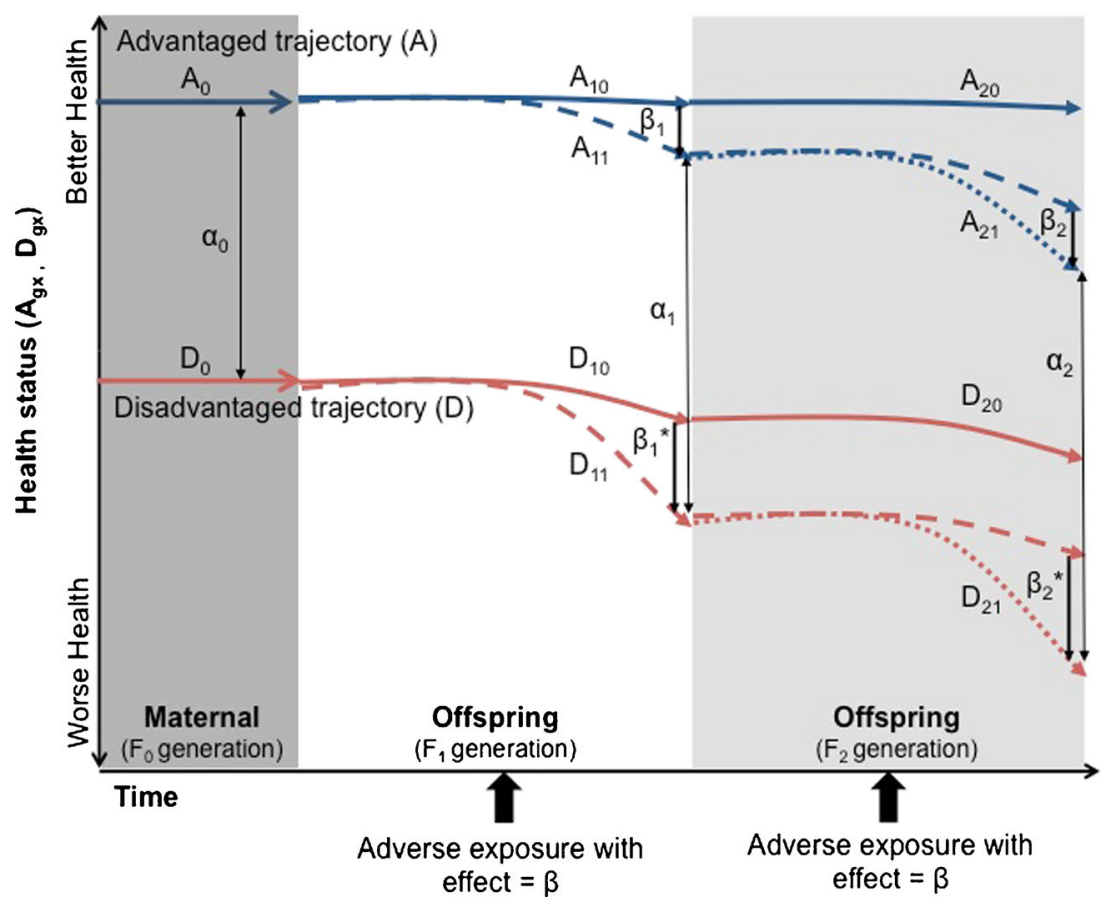


transmission of poverty, low educational expectations, and unhealthy coping behaviors.

One important element of Fig. 1 is that the effect $\beta$ is not constant but instead varies according to exposure status in the previous generation. This effect difference, by advantaged or disadvantaged trajectories $\left(\mathrm{A}_{\mathrm{g} 1}\right.$ minus $\mathrm{A}_{\mathrm{g} 0}$ or $\mathrm{D}_{\mathrm{g} 1}$ minus $\mathrm{D}_{\mathrm{g} 0}$ ), is illustrated by comparing the exposure effects $\beta_{1}$ versus $\beta_{1}$ * and $\beta_{2}$ versus $\beta_{2}{ }^{*}$. The magnitude of effect, $\beta$, may be influenced by the biological processes underlying developmental programming. That is, maternal prenatal exposures may alter the physiology of the gestating offspring such that the offspring is more sensitive to the effects of postnatal exposures such as environmental pollutants, stress, or high-fat diet.

Postnatal exposures, referred to above as ongoing adverse exposures, comprise second hits to biological predispositions determined during gestation. We propose that this heightened sensitivity is a mechanism through which developmental programming exacerbates health disparities across generations. The increasing magnitude of disparity that results from differential exposure effects can be seen by comparing $\alpha_{1}$ with $\alpha_{2}$. Not only are women in disadvantaged trajectories at increased risk of poor health in the absence of ongoing exposures, they, and by extension their offspring, may experience a greater health "penalty" to ongoing postnatal insults. By contrast, advantaged offspring will be less likely to experience detrimental exposures later in life but, more importantly, will be more resilient than their less advantaged counterparts. In this review, we focus on evidence that effects of key postnatal exposures differ according to maternal exposure status $(\beta$ versus $\left.\beta^{*}\right)$. One enduring question in the social epidemiologic literature is why health disparities persist in light of targeted health promotion endeavors. One part of the health disparities' intransigence may be that disadvantaged populations produce a larger than expected response to ongoing socialenvironmental insults in part, because they have been biologically primed to do so.

\section{Objectives}

We first review evidence that maternal exposure to air pollution, psychosocial stress, and overnutrition induces exaggerated offspring responses to subsequent social environmental exposures. We then discuss the social patterning of social environmental stressors and how each type of gestational exposure may contribute to the construction and transmission of health disparities. While existing reviews describe how gestational exposures impact offspring health (e.g., [16, 17, 19]), this paper focuses on how developmental programming can exacerbate the effects of socially patterned adverse environmental conditions, like those studied by social epidemiologists.

\section{Methods}

We performed an electronic search of publications on Google Scholar, PubMED, and EBSCO Host databases. The following search terms were used: "fetal priming," "fetal exposures," "fetal programming," "early life exposures," "developmental origins," and "intergenerational," in combination with a second group of key words such as, "adult health outcomes," "adult health," "offspring," "influences on health," "disease in later life," "second hit," and "child development." To search for relevant papers in domains of interest, we included additional terms including "air pollution" and "stress"; For maternal overnutrition exposures, we searched for (obesity or "high-fat" or "fat") and (maternal or gestation or pregnancy) and (offspring or fetal). In addition to using key word combinations, we employed the "related articles" electronic prompts provided with the search results and examined references from relevant papers. We retained original human and animal studies that included a gestational exposure and a childhood or adulthood second hit exposure. While the papers presented in the following tables do not represent the universe of possible manuscripts, they are representative of the second hit literature as of August 2014. This review does not discuss fetal outcomes resulting from gestational exposures, even though this work is relevant to future adult health.

\section{Results}

\section{Environmental Pollutants}

Poor environmental quality, like other social epidemiologic phenomena, has its greatest impact on those whose health status is already at risk [20], such as individuals living on the disadvantaged trajectory in our conceptual model. Exposure to environmental pollutants, inadequate buffering of environmental exposures, and increased susceptibility to environment-induced poor health owing to existing comorbidities are differentially distributed [21-24]. We propose developmental programming as an additional explanation for the disparate effects of environmental exposures by considering the evidence that gestational exposures to air pollution predispose offspring to worse physiological responses to subsequent air pollution experiences.

Evidence from animal studies suggests that exposure to air pollutants by neonates (e.g., mouse pups) during gestation is associated with an exaggerated reaction to air pollution exposure during child- or adulthood (Table 1). In studies by Auten and colleagues $[25 \bullet \cdot, 26]$, pups born to moms who were exposed to diesel exhaust while gestating, who were then exposed to ozone during their childhood, developed persistent airway hyper-reactivity. Effects of childhood ozone exposure were stronger in pups born to dams exposed to diesel exhaust 
while gestating, than in pups born to dams that were instead exposed to filtered air [25••]. This paper follows earlier work, which reported that maternal diesel exposure induced inflammatory cytokine responses in the offspring that presumably contribute enhanced airway hyper-responsiveness to postnatal ozone exposure [26].

A handful of observational epidemiologic studies examining gestational and later-life exposures investigated if fetal priming of later response is generalizable to humans. Perera [27] and colleagues collected polycyclic aromatic hydrocarbon (PAH) data using a personal air monitor during the third trimester of pregnancy. Postnatal interviews were administered at 6 months postdelivery and annually thereafter to determine changes in PAH exposure. Youth exposed to high amounts of PAH in utero exhibited lower intelligence quotient scores, but postnatal exposure did not exacerbate this effect. A second epidemiologic effort to tease apart effects of gestational and adult exposures comes from Montgomery and Ekbom [28]. They considered maternal smoking, a form of gestational air pollution, and the offspring's own smoking at age 16 as the second air pollution hit related to diabetes development. After adjustment for maternal smoking, cigarette smoking during adolescence was independently associated with increased risk of diabetes. No evidence of hyper-sensitization was observed, but the authors went on to suggest mechanisms by which this observed effect is biologically plausible.

While the number of studies considering gestational air pollution exposures followed by subsequent air pollution is relatively small, the results suggest that gestational exposure primes the child or adult body for a more severe reaction to subsequent exposures. This finding is likely to be highly relevant for human health given the ubiquity of air pollution. Further, because social stratification results in non-randomly distributed adverse air quality, those most vulnerable to the effects of poor air quality are most likely ensured of ongoing adverse exposures.

\section{Psychosocial Stress}

Lack of autonomy within a social hierarchy, inadequate income, discrimination, and inadequacy of time and resources are sources of chronic psychosocial stress in disadvantaged populations and are linked with adverse health outcomes. Maternal stress induces elevations in the glucocorticoid hormone, cortisol [29]. Excessive maternal cortisol can overwhelm placental capacity to block its passage; fetal exposure to cortisol induces widespread developmental alterations that increase susceptibility to disease [12]. Notably, glucocorticoids are a common pathway through which a wide range of adverse maternal exposures induces developmental programming [30]. We reviewed evidence that maternal stress during pregnancy induces exaggerated response to stress experienced by the offspring.
In animal studies, offspring born to dams exposed to restraint stress, who then experienced stress during adolescence, have altered immune, neuroendocrine, or glucocorticoid systems, compared to those animals not exposed to prenatal maternal restraint (Table 2). Pascuan and colleagues [31] found that prenatal stress altered the intrinsic regulation response to acute stress in adult mice, while $\mathrm{Xu}$ and colleagues [32] found that prenatal restraint of pregnant rats led to a hyper-responsiveness of the HPA axis, and increased anxiety-like behavior in rats. Van den Hove and colleagues found that, compared with control rats, chronic variable mild stress in adolescent rats that had been exposed to maternal restraint stress during gestation partially offset the effects of prenatal stress, suggesting that responses to developmental stress may depend on later life exposures [33••]. In contrast, Schopper and colleagues [34] showed that intermittent light stress induced elevated glucocorticoid levels in maternal guinea pigs but that their offspring had an attenuated cortisol response over adult development and subsequent stress exposures.

Human evidence for the effect of second hit stress exposures following prenatal stress is scant. One study collected the peripheral blood mononuclear cells from 34 young women whose mothers reported negative life events during their pregnancy (and from a comparison group whose mothers did not experience negative life events). The cytokine response to a potent $\mathrm{T}$ cell stimulator, or a phytohemagglutinin (PHA) second hit, was significantly altered in women born to mothers who were stressed while gestating [35], suggesting that prenatal stress exposure may induce an exaggerated immune response in adult women. Regardless of the exact source of social stress, maternal stress while gestating likely induces physiologic changes in the developing fetus, such that subsequent postnatal (lifetime) stress elicits perturbations in the offspring's stress response.

\section{Nutrition}

"Western-style" diets, characterized by high fat and high refined sugar content, are ubiquitous in Western populations and even more pronounced in racial minority and low-income populations [36, 37]. The racial and neighborhood-level patterning of poor-quality food environments is a significant area of social epidemiologic research. We reviewed evidence for the hypothesis that prenatal Western-style diet (high fat, high sugar, or high-total energy) induces exaggerated offspring responses to Western-style diet in postnatal life (Table 3). A growing body of animal research supports this hypothesis. For example, Bruce and colleagues examined the combined effects of maternal high-fat diet during preconception, gestation, and lactation in mice and high-fat diet consumed by the offspring upon weaning [38]. Postweaning high-fat diet induced accelerated weight gain, higher adiposity, cholesterol 


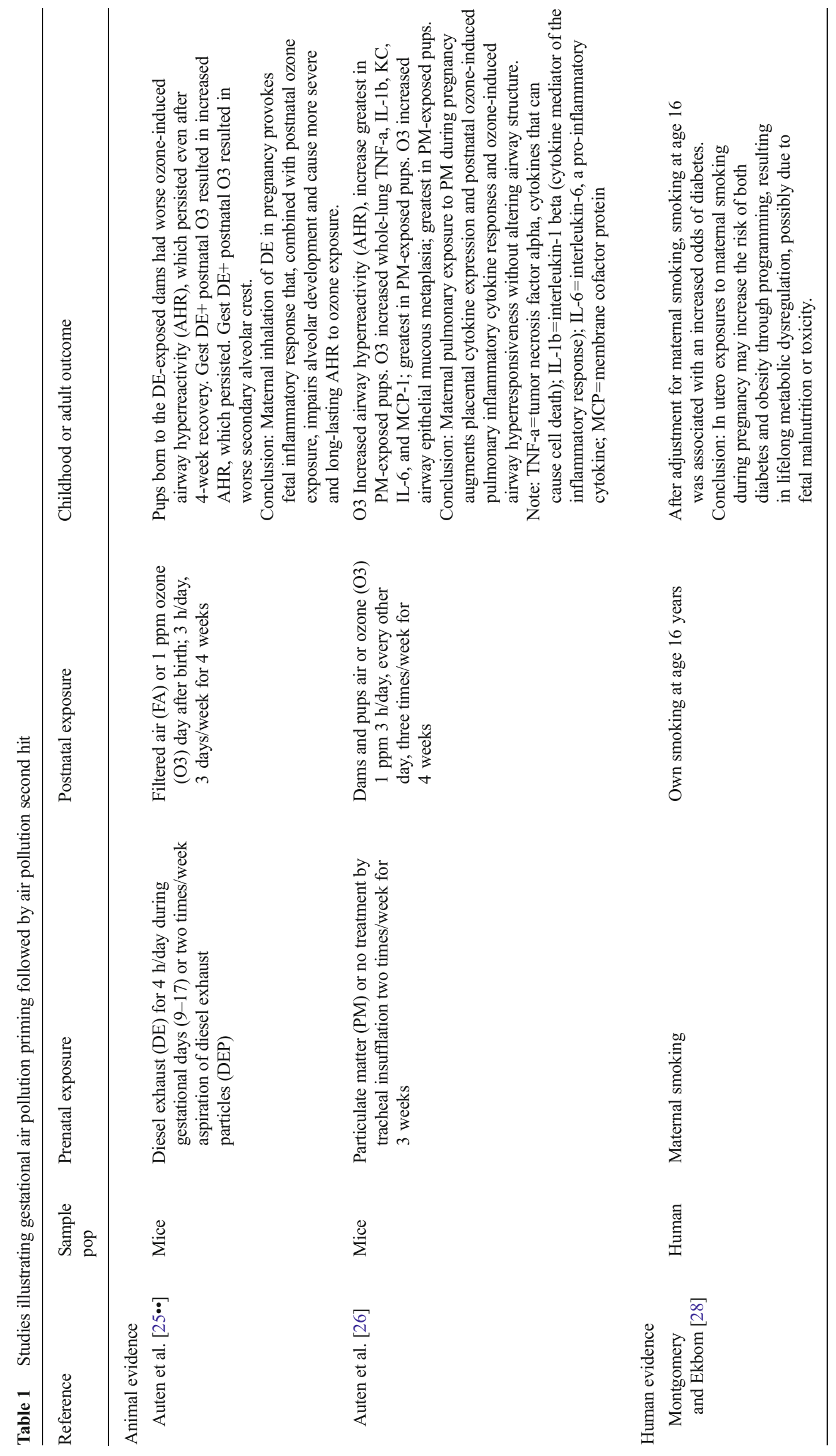




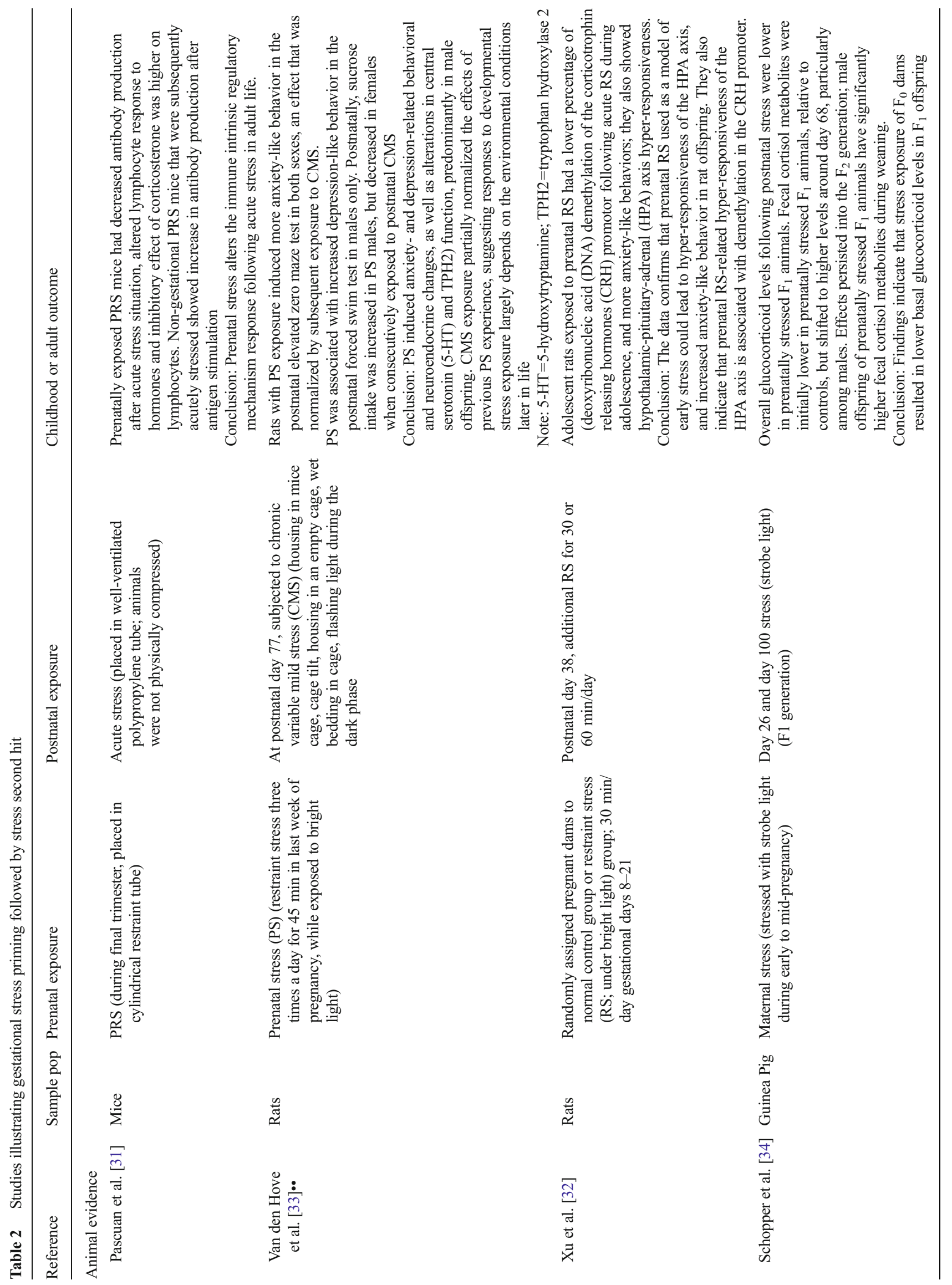




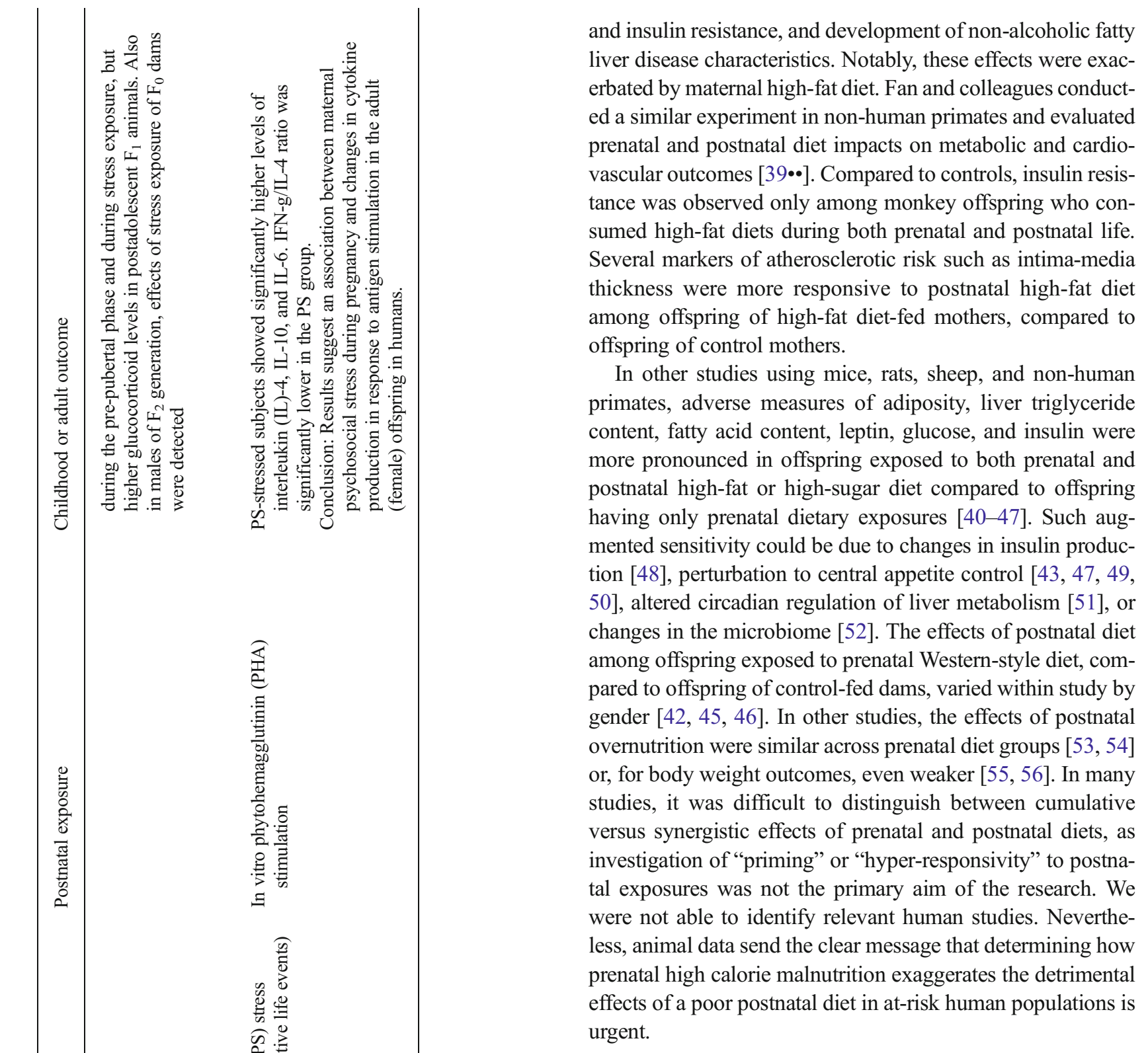

Cross-Domain Exposure Effects

Humans live in environments characterized by simultaneous exposures from multiple domains, each of which are socially structured and would likely exacerbate existing health disparities. The animal literature has begun to replicate this exposure complexity by conducting cross-exposure experiments (Table 4). Recent research by Wei and colleagues exemplifies this work. Wei [57] found that prenatal exposure to bisphenol A, a synthetic compound with hormone-like properties commonly employed in plastics production, resulted in defective insulin signaling among offspring exposed to standard diets. However, compared to unexposed controls, the prenatally exposed BPA pups developed more extensive liver impairment (e.g., increased inflammation and mild fibrosis) following exposure to high-fat diets. The authors concluded that 


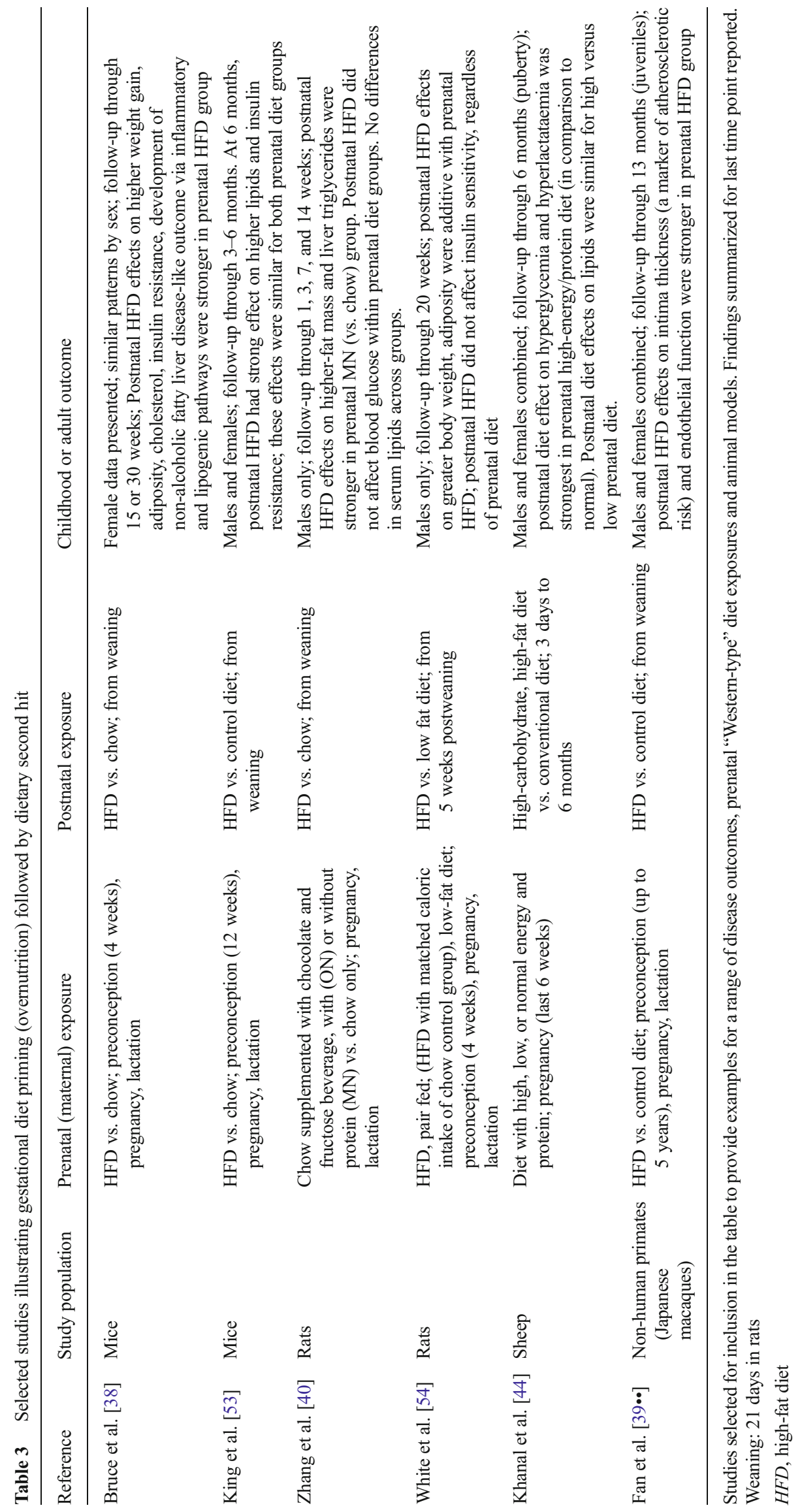




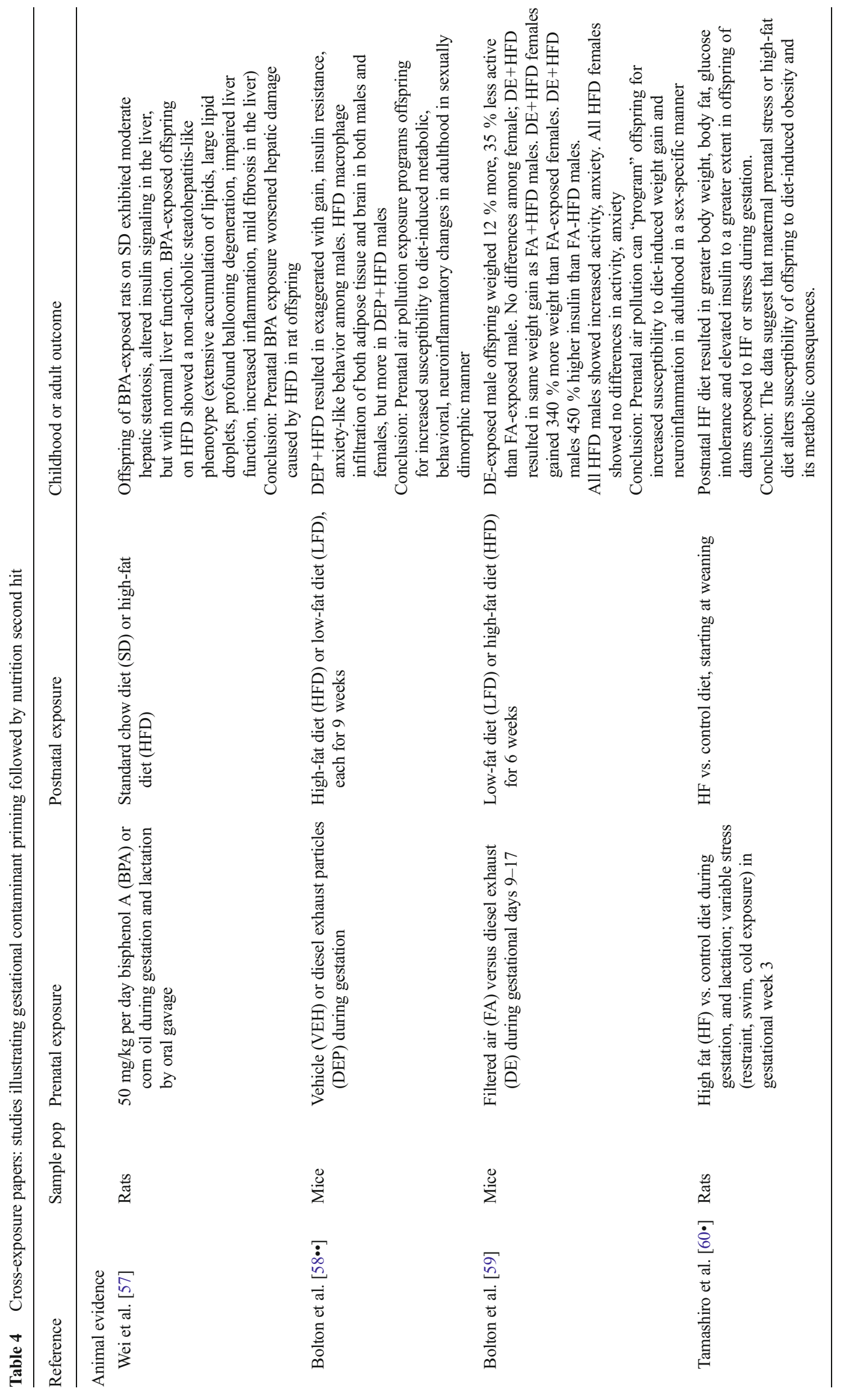


prenatal BPA exposure predisposed offspring who later consumed high-fat diets to develop metabolic syndrome manifestations.

In 2014, Bolton and colleagues [58••] reported that prenatal diesel exhaust exposure, followed by a postnatal high fat (compared to a low fat) dietary second hit, programmed offspring for diet-induced metabolic, behavioral, and neuroinflammatory changes in adulthood and that these changes were greatest among males. This work followed their prior report [59] that prenatal exposure to diesel exhaust followed postnatally by high-fat diet programmed offspring for weight gain and neuro-inflammation in adulthood. These findings, too, differed by sex: postnatal high-fat diet induced $340 \%$ more weight gain in females with prenatal diesel exposure than females with prenatal filtered air exposure, while postnatal high-fat diet induced $450 \%$ higher insulin in prenatal diesel-exposed males, compared to prenatal filtered airexposed males.

Another example illustrates the potential cumulative effects of multiple, cross-domain prenatal insults. Tamashiro and colleagues conducted a unique experiment in rats, in which dams were exposed to high-fat diet, an 8-day variable stress protocol, a combination of the two, or control conditions during pregnancy $\left[60^{\bullet}\right]$. Offspring were fed either a control or high-fat diet upon weaning. The postweaning high-fat diet induced greater weight gain and, among male offspring, lower glucose tolerance than in controls.

Humans living on the disadvantaged trajectory are most likely simultaneously exposed to poor air quality, high stress, and nutrition-poor food environments. Social epidemiologic research suggests that barring substantial intervention, residents in disadvantaged areas are likely to remain there. Therefore, these cross-domain exposure effects have profound implications for social epidemiologic research and the construction of persistent health disparities.

\section{Discussion}

The mechanisms by which health disparities propagate through generations are not well understood. In this article, we considered developmental programming as a possible contributor to persistent health disparities. We reviewed published research in three prenatal and postnatal exposure domains: air pollution, psychosocial stress, and overnutrition. A growing body of evidence supports the hypothesis that prenatal exposures to environmental stressors prime offspring for exaggerated response to childhood or adulthood exposures. While early in its development, these findings are similar across species, including a small number of observational human studies, as well as across domains. Taken together, this research justifies further consideration of how developmental programming may contribute to constructing and exacerbating health disparities across generations.

\section{Effect Modification of Established Social Processes by Developmental Priming Status}

Health is propagated across generations through multiple pathways, including genetics, behavioral modeling and literacy, and environmental continuity. We propose developmental programming not as a competing or alternate theory of intergenerational transmission but rather as a previously unappreciated process that can magnify the effects of social epidemiologic intergenerational processes. While genetic susceptibility puts some individuals at higher risk of specific health conditions, it rarely varies by race $[61,62]$ and is not modifiable, so will not be discussed further in this review.

In our conceptual model (Fig. 1), health disparities are exacerbated from one generation to the next as a result of (a) the effects of suboptimal gestational environments on exaggerated offspring response to subsequent environmental and social exposures $\left(\beta_{1}\right.$ versus $\beta_{1}{ }^{*}$ and $\beta_{2}$ versus $\left.\beta_{2}{ }^{*}\right)$ and (b) intergenerational continuity of adverse exposures (prevalence of $\mathrm{A}_{\mathrm{g} 0}, \mathrm{~A}_{\mathrm{g} 1}, \mathrm{D}_{\mathrm{g} 0}, \mathrm{D}_{\mathrm{g} 1}$ scenarios). Vast social epidemiologic evidence supports the continuity of adverse exposures, perhaps most thoroughly for residential conditions. Racial residential segregation results in the geographic clustering of nonwhite or non-wealthy individuals in the USA [63]. Environmental disamenities are also non-randomly distributed [64] and cluster geographically, particularly in low-wealth, nonwhite neighborhoods. This is true for sources of air pollution $[65,66]$, crime, environmental sources of psychosocial stress such as graffiti and social disorder [67-70], liquor stores [71, 72], unhealthy food environments [73-76], and inadequate physical activity resources [77-79]. Social epidemiologic research consistently indicates that residence in close proximity to negative environmental conditions is associated with increased risk of a variety of poor health outcomes [80-82].

Other mechanisms that contribute to the inequitable allocation of chronic, adverse exposures have been proposed; their effects could also be modified by an individual's developmental priming status. Contemporary social conditions deriving from historic injustices, including subjugation (slavery for African Americans and displacement of Native Americans) $[83,84]$ and ongoing racial/ethnic discrimination [85-87] contribute to chronic social stress and have been considered in the social epidemiologic literature. Intergenerational poverty $[88,89]$ and parental modeling of health-related behavior [90] have also been implicated in poor offspring health outcomes and are among the possible pathways through which health disparities are propagated.

The implications of the combined gestational priming plus differential distribution of adverse exposures is that residents of suboptimal environments will have both poorer health at 
baseline and a propensity for an exaggerated negative health response to subsequent adverse exposures, which are differentially distributed throughout the next generation's life course. It suggests that developmental priming processes and social epidemiologic exposures interact to socially structure health states.

\section{Social Epidemiologic Research Gaps}

Our proposed theory of the contribution of developmental programming to intergenerational health disparities is based upon empirical evidence in our literature review and decades of social epidemiologic research. Yet, research on the intersection between developmental programming and health disparities is scant.

A handful of studies suggest differences in methylation patterns according to social factors [91, 92], but this area of study is in its infancy. Another critical component in testing our theory is investigation of the extent to which developmental programming biomarkers explain differences in health and responsivity to exposures in advantaged versus disadvantaged groups. Such differences likely vary according to disease outcome, specific social groups, and life stage.

Inclusion of developmental programming biomarkers would help to overcome limitations stemming from the vast number of factors that are entangled with social disadvantage. However, key barriers to this line of inquiry are the still growing knowledge about methylation sites and other biomarkers relevant to specific disease processes. Such knowledge is critical for linking intergenerational health disparities with mechanisms identified in biological developmental programming research.

\section{Social Epidemiologic Research Challenges}

Investigation of developmental programming as a contributor to health disparities requires observational research on socially patterned exposures and outcomes in human study populations. Such epidemiologic research poses several methodological challenges.

Resilience Not every person whose mother is exposed to poor air quality goes on to develop airway hyperreactivity; nor does every person whose mother was obese during pregnancy become obese him/herself. The presence of resilience, or the ability to properly adapt to stress and adversity, is either a robust homeostasis-supporting biologic phenomenon or it constitutes counter-evidence for developmental programming effects. Research that carefully assesses prenatal and postnatal exposure from a developmental priming perspective, with special attention to resilience and the factors that seem to support healthy coping, will help quantify what portion of health outcomes, if any, might be attributable to programming effects.
Long-Term, Intergenerational Follow-Up Our most robust understanding of developmental programming examines intergenerational effects of experimentally assigned maternal exposures. Adverse offspring later-life effects are often observed even when the gestationally exposed and non-exposed are similar (in their size, other biomarkers) at birth. Therefore, the indicators of adverse fetal development (e.g., birth weight) typically used in epidemiologic studies are inadequate as measures of adverse developmental programming. Rather, measurement of developmental programming requires data collection on both mothers and offspring, in study populations large enough to test prenatal and postnatal interactions. Research needs will include the use of data linkage, improved retrospective maternal health status assessment, and intergenerational follow-up. Studying priming effects or second hits in humans, even observationally, will require long-term cohort construction and funding programs that endure across generations.

Within-Person, Within-Family Variability Many of the exposures that we know contribute to fetal priming are ubiquitous (e.g., social stress) and have become part of our daily environment (e.g., diesel exposure). As a result, constructing unexposed groups for causal comparisons is challenging. In addition, it is difficult to disentangle maternal effects (e.g., maternal high-fat diet) from offspring effects (e.g., ongoing offspring high-fat diet). Existing human research studying prenatal/postnatal interactions typically rely on short-term experimental interventions such as a short period of overfeeding or inactivity [93] to attain variability in postnatal exposures. However, such effects are unlikely to be comparable with the chronic exposures humans experience. Social epidemiologic research of developmental priming may require large, cross-national studies to construct adequate exposure variability to observe intergenerational effects.

Complexity Our review suggests that multiple, interrelated and socially patterned prenatal and postnatal exposures contribute to intergenerational disparities. The inclusion of developmental programming into social epidemiologic models introduces additional complexity to etiologic pathways for understanding health disparities. For example, maternal overnutrition leads to neurological rewiring of appetite circuits and the propensity to gain weight among offspring; thus elevated appetite is a partial mediator in the pathway from developmental programming to obesity. The investigation of developmental programming as both a mediator and a moderator of typical social epidemiologic exposures requires innovative analytic approaches such as emerging decomposition methods [94]. Furthermore, these second hits may have lagged effects within the life cycle or across generations and may induce differential effects according to life stage. Examination of these types of complexities requires approaches such as structural equation modeling (SEM) [95] or complex systems modeling [96]. 
Translation for Public Health Policy

The reduction or elimination of health disparities is a national priority named by many health-related agencies, including the Centers for Disease Control and Prevention, Healthy People 2020 and Agency for Healthcare Research and Quality. Despite resources devoted to this endeavor, health disparities persist. The evidence reported here, which developmental programming primes subsequent generational responses to ubiquitous social-epidemiologic exposures, introduces important considerations for efforts aimed at reducing health disparities.

Targeted Exposure and Intervention Effects In this review, we provide evidence that postnatal exposures in three domainsair pollution, psychosocial stress, and overnutrition — result in stronger negative effects among prenatally exposed individuals (Fig. 1: $\beta$ versus $\beta^{*}$ ). Left unaddressed, poor health outcomes associated with these exposures are likely to exacerbate existing health disparities through developmental programming and social patterning mechanisms. Conversely, interventions targeting these exposures among the most vulnerable populations have the potential to exert powerful, positive effects (Fig. 2: $\alpha_{1}{ }^{*}$ versus $\alpha_{2}{ }^{*}$ ).

Environmental Remediation and Enrichment The research reviewed here suggests that biological predispositions for adverse health effects may be established during gestation through developmental programming or priming. For priming to exert an adverse effect, however, it requires a second adverse environmental exposure for a predisposition to become actualized. Therefore, environmental enrichment represents a potent tool for improving health trajectories (Fig. 2: $\alpha_{1} *$ versus $\alpha_{2}^{*}$ ). While we cannot undo the priming that has already occurred, we can reduce its ultimate effects by restricting the number and nature of second insults a given primed individual receives. Public policy and industrial regulation can be used to improve air quality while economic and cultural reform could reduce social stressors by socially and financially supporting disadvantaged populations. Changing social environments is one of the most effective routes to affecting population prevalence of both risk factors and disease outcomes [97]. Likewise, long-term improvements in dietary practices will likely require policy changes targeting food availability, affordability, and culture [98]. Addressing these contextual exposures is undoubtedly challenging; yet evidence suggests that altering environmental exposures is one of the few interventions that can be used to improve health for the most vulnerable groups. For instance, environmental enrichment following lead exposure among children has been associated with cognitive improvements [99]. The research presented here also suggests that environmental remediation will not only reduce the magnitude and/or likelihood of the second hit, it will also reduce the priming effect for the next generation.

Expanded Time Frame While national public health objectives seek to eliminate health disparities by 2020 , our conceptual model illustrates the long period of time that may be required to ameliorate them, even with dramatic and sustained reduction in exposure prevalence. The magnitude of risk transmission from one generation to the next is unknown. Quantification of the intergenerational transmission rate, as called for under Resilience above, would improve our ability to redefine the time frame in which elimination of health disparities is possible.
Fig. 2 Theoretical role of the relationship between environmental enrichment and developmental programming on reducing health disparities over three generations. $A_{g x}$ and $D_{g x}$ denote health status $Y_{g x}$ for generation $g$ and exposure status x. $\alpha_{g}$ denotes the magnitude of disparity in any in generation $\mathrm{g}$

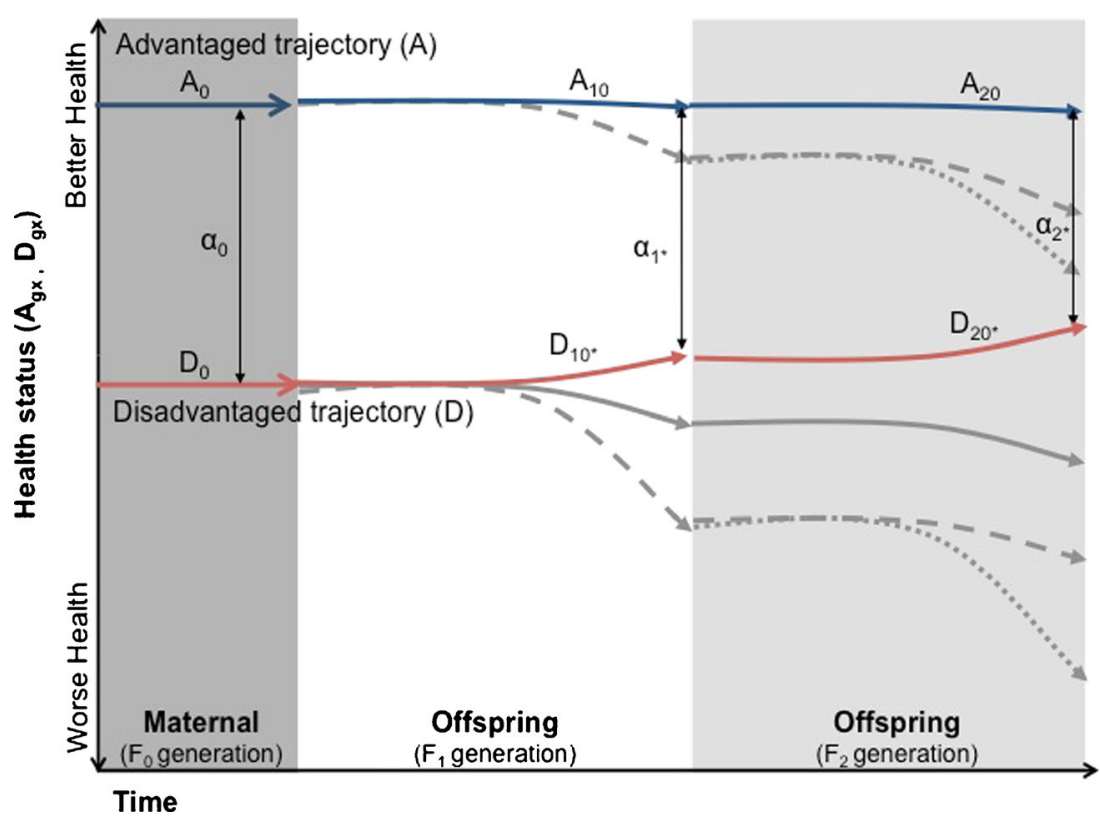


With multicomponent interventions that target both environmental and behavioral objectives thought to most potently reduce adverse developmental programming, we should seek to attain incremental improvements in health from one generation to the next (e.g., the health improvement from $\mathrm{D}_{0}$ to $\mathrm{D}_{10}$, and $D_{10}$ to $D_{20}$ in Fig. 2). Identification of such targets is needed and will require interdisciplinary research and programs of broad scope.

\section{Conclusion}

Public health scholars, practitioners, and policymakers have called for an explicit examination of drivers of disparities. Our conceptual framework suggests that this is even more important due effect magnification of social epidemiologic exposures by developmental programming. Awareness of key developmental programming processes provides an opportunity to identify high-leverage factors for reducing persistent disparities. It also affirms that environmental modification - providing healthy and safe environments in which to live, work, and play - is likely the single best intervention to ensure health for all.

\section{Compliance with Ethics Guidelines}

Conflict of Interest LC Messer, J Boone-Heinonen, L Mponwane, L Wallack, and KL Thornburg all declare no conflicts of interest.

Human and Animal Rights and Informed Consent This article does not contain any studies with human or animal subjects performed by any of the authors.

\section{References}

Papers of particular interest, published recently, have been highlighted as:

-. Of major importance

- Of importance

1. Bayman E, Drake A, Piyasena C. Prematurity and programming of cardiovascular disease risk: a future challenge for public health? Archives of disease in childhood. Fetal Neonatal Ed. 2014;99(6): F510-4.

2. Wells J. Adaptive variability in the duration of critical windows of plasticity: implications for the programming of obesity. Evol Med Pub Health. 2014;2014(1):109-21.

3. Alexander B, Henry Dasinger J, Intapad S. Effect of Low birth weight on women's health. Clin Ther. 2014;S0149-2918(14): 00390-7.

4. Kuzawa C, Sweet E. Epigenetics and the embodiment of race: developmental origins of US racial disparities in cardiovascular health. Am J Hum Biol. 2009;21(1):2-15.
5. Silva L et al. Mother's educational level and fetal growth: the genesis of health inequalities. Int J Epidemiol. 2010;39(5):1250 61.

6. Jasienska G. Low birth weight of contemporary African Americans: an intergenerational effect of slavery? Am J Hum Biol. 2009;21(1):16-24.

7. Thayer ZM, Kuzawa C. Biological memories of past environments: epigenetic pathways to health disparities. Epigenetics. 2011;6(7): 798-803.

8. Thornburg KL et al. In utero life and epigenetic predisposition for disease. Adv Genet. 2010;71:57-78.

9. Thornburg K, O'Tierney P, Louey S. Review: the placenta is a programming agent for cardiovascular disease. Placenta, 2010(31 Suppl): p. S54-S59.

10. Wright R, Brunst K. Programming of respiratory health in childhood: influence of outdoor air pollution. Curr Opin Pediatr. 2013;25(2):232-9.

11. Rauh $\mathrm{V}$ et al. Neonatology and the environment: impact of early exposure to airborne environmental toxicants on infant and child neurodevelopment. Neoreviews. 2010;11:363-9.

12. Entringer $\mathrm{S}$ et al. Fetal programming of body composition, obesity, and metabolic function: the role of intrauterine stress and stress biology. J Nutr Metab. 2012;2012:632548.

13. Brunton P. Resetting the dynamic range of hypothalamic-pituitaryadrenal axis stress responses through pregnancy. J Neuroendocrinol. 2010;22(11):1198-213.

14. Dabelea D, Crume T. Maternal environment and the transgenerational cycle of obesity and diabetes. Diabetes. 2011;60(7):1849-55.

15. Ainge $\mathrm{H}$ et al. A systematic review on animal models of maternal high fat feeding and offspring glycaemic control. Int $\mathrm{J}$ Obes. 2011;35(3):325-35.

16. Gluckman $\mathrm{P}$ et al. Effect of in utero and early-life conditions on adult health and disease. N Engl J Med. 2008;359(1):61-73.

17. Burdge G, Lillycrop K. Nutrition, epigenetics, and developmental plasticity: implications for understanding human disease. Annu Rev Nutr. 2010;30:315-39.

18. Geronimus A. The weathering hypothesis and the health of AfricanAmerican women and infants: evidence and speculations. Ethn Dis. 1992;2:207-21.

19. McMillen I, Robinson J. Developmental origins of the metabolic syndrome: prediction, plasticity, and programming. Physiol Rev. 2005;85(2):571-633.

20. Gee G, Payne-Sturges D. Environmental health disparities: a framework integrating psychosocial and environmental concepts. Environ Health Perspect. 2004;112(17):1645-53.

21. O'Neill $\mathrm{M}$ et al. Health, wealth, and air pollution: advancing theory and methods. Environ Health Perspect. 2003;111(16):1861-70.

22. Jerrett M, et al. What GIS tells us about environmental and public health. 2009.

23. Miranda $\mathrm{M}$ et al. Making the environmental justice grade: the relative burden of air pollution exposure in the United States. Int J Environ Res Publ Health. 2011;8(6):1755-71.

24. Bell M, Ebisu K. Environmental inequality in exposures to airborne particulate matter components in the United States. Environ Health Perspect. 2012;120(12):1699-704.

25.• Auten RL et al. Maternal diesel inhalation increases airway hyperreactivity in ozone-exposed offspring. Am J Respir Cell Mol Biol. 2012;46(4):454-60. This paper demonstrates how prenatal exposure primes for an exaggerated response to postnatal exposure.

26. Auten RL et al. Maternal exposure to particulate matter increases postnatal ozone-induced airway hyperreactivity in juvenile mice. Am J Respir Crit Care Med. 2009;180(12):1218-26.

27. Perera FP et al. Prenatal airborne polycyclic aromatic hydrocarbon exposure and child IQ at age 5 years. Pediatrics. 2009;124(2):e195202. 
28. Montgomery S, Ekbom A. Smoking during pregnancy and diabetes mellitus in a British longitudinal birth cohort. Br Med J. 2002;324(7328):26-7.

29. Djuric $Z$ et al. Biomarkers of psychological stress in health disparities research. Open Biomark J. 2008;1:7-19.

30. Reynolds R. Glucocorticoid excess and the developmental origins of disease: two decades of testing the hypothesis-2012 Curt Richter Award Winner. Psychoneuroendocrinology. 2013;38(1):111.

31. Pascuan CG et al. Prenatal stress induces up-regulation of glucocorticoid receptors on lymphoid cells modifying the T-cell response after acute stress exposure in the adult life. Physiol Behav. 2014;128:141-7.

32. $\mathrm{Xu} \mathrm{L}$ et al. Prenatal restraint stress is associated with demethylation of corticotrophin releasing hormone $(\mathrm{CRH})$ promoter and enhances CRH transcriptional responses to stress in adolescent rats. Neurochem Res. 2014;39(7):1193-8.

33.• Van den Hove DL et al. Prenatal stress and subsequent exposure to chronic mild stress in rats; interdependent effects on emotional behavior and the serotonergic system. Eur Neuropsychopharmacol. 2014;24(4):595-607. This paper is important because it demonstrates how responses to developmental stress exposures depend on later life environmental conditions; this suggests modifying later life environments may help overcome early life adverse exposures.

34. Schopper H et al. Effects of prenatal stress on hypothalamicpituitary-adrenal (HPA) axis function over two generations of guinea pigs (Cavia aperea f. porcellus). Gen Comp Endocrinol. 2012;176(1):18-27.

35. Entringer $\mathrm{S}$ et al. Influence of prenatal psychosocial stress on cytokine production in adult women. Dev Psychobiol. 2008;50(6):579-87.

36. Thompson $\mathrm{F}$ et al. Interrelationships of added sugars intake, socioeconomic status, and race/ethnicity in adults in the United States: National Health Interview Survey, 2005. J Am Diet Assoc. 2009;109(8):1376-83.

37. Kirkpatrick $\mathrm{S}$ et al. Income and race/ethnicity are associated with adherence to food-based dietary guidance among US adults and children. J Acad Nutr Diet. 2012;112(5):624-35.

38. Bruce $\mathrm{K}$ et al. Maternal high-fat feeding primes steatohepatitis in adult mice offspring, involving mitochondrial dysfunction and altered lipogenesis gene expression. Hepatology. 2009;50(6):1796808.

39.• Fan L et al. Maternal high-fat diet impacts endothelial function in nonhuman primate offspring. Int J Obes. 2013;37(2):254-62. This paper is reports results for non-human primates (closest to humans) and provides a nice study design.

40. Zhang $Z$ et al. Supplementation of the maternal diet during pregnancy with chocolate and fructose interacts with the high-fat diet of the young to facilitate the onset of metabolic disorders in rat offspring. Clin Exp Pharmacol Physiol. 2013;40(9):652-61.

41. Pruis $\mathrm{M}$ et al. Maternal western diet primes non-alcoholic fatty liver disease in adult mouse offspring. Acta Physiol (Oxf). 2014;210(1): 215-27.

42. Bayol S et al. A maternal "junk food" diet in pregnancy and lactation promotes nonalcoholic fatty liver disease in rat offspring. Endocrinology. 2010;151(4):1451-61.

43. Chen H, Simar D, Morris M. Hypothalamic neuroendocrine circuitry is programmed by maternal obesity: interaction with postnatal nutritional environment. PLoS ONE. 2009;4(7):e6259.

44. Khanal P, et al. Late gestation under- and overnutrition have differential impacts when combined with a postnatal obesogenic diet on glucose-lactate-insulin adaptations during metabolic challenges in adolescent sheep. Acta Physiologica (Oxf), 2014.

45. Mitra A et al. Effect of high-fat diet during gestation, lactation, or postweaning on physiological and behavioral indexes in borderline hypertensive rats. Am J Physiol Regul Integr Comp Physiol. 2009;296(1):R20-8.

46. Shankar K et al. Maternal obesity at conception programs obesity in the offspring. Am J Physiol Regul Integr Comp Physiol. 2008;294(2):R528-38.

47. Page $\mathrm{K}$ et al. Maternal and postweaning diet interaction alters hypothalamic gene expression and modulates response to a highfat diet in male offspring. Am J Physiol Regul Integr Comp Physiol. 2009;297(4):R1049-57.

48. Catalano P et al. Fetuses of obese mothers develop insulin resistance in utero. Diabetes Care. 2009;32(6):1076-80.

49. Grove K et al. Development of metabolic systems. Physiol Behav. 2005;86(5):646-60.

50. Samuelsson A et al. Diet-induced obesity in female mice leads to offspring hyperphagia, adiposity, hypertension, and insulin resistance: a novel murine model of developmental programming. Hypertension. 2008;51(2):383-92.

51. Borengasser $\mathrm{S}$ et al. High fat diet and in utero exposure to maternal obesity disrupts circadian rhythm and leads to metabolic programming of liver in rat offspring. PLoS ONE. 2014;9(1):e84209.

52. Ma J et al. High-fat maternal diet during pregnancy persistently alters the offspring microbiome in a primate model. Nat Commun. 2014;5:3889.

53. King V et al. Post-weaning diet determines metabolic risk in mice exposed to overnutrition in early life. Reprod Biol Endocrinol. 2014;12:73.

54. White C, Purpera M, Morrison C. Maternal obesity is necessary for programming effect of high-fat diet on offspring. Am J Physiol Regul Integr Comp Physiol. 2009;296(5):R1464-72.

55. Couvreur $\mathrm{O}$ et al. Unexpected long-term protection of adult offspring born to high-fat fed dams against obesity induced by a sucrose-rich diet. PLoS ONE. 2011;6(3):e18043.

56. Fereaou-Viala $J$ et al. Long-term consequences of maternal high-fat feeding on hypothalamic leptin sensitivity and diet-induced obesity in the offspring. Am J Physiol Regul Integr Comp Physiol. 2007;293(3):R1056-62.

57. Wei J et al. Perinatal exposure to bisphenol A exacerbates nonalcoholic steatohepatitis-like phenotype in male rat offspring fed on a high-fat diet. J Endocrinol. 2014;222(3):313-25.

58.• Bolton JL, Auten RL, Bilbo SD. Prenatal air pollution exposure induces sexually dimorphic fetal programming of metabolic and neuroinflammatory outcomes in adult offspring. Brain Behav Immun. 2014;37:30-44. This paper is useful in providing mixed domain prenatal (air) and postnatal (diet) exposures, which better replicates the human experience. It also demonstrated sexuallydimorphic effects, which is what we might expect.

59. Bolton JL et al. Prenatal air pollution exposure induces neuroinflammation and predisposes offspring to weight gain in adulthood in a sex-specific manner. FASEB J. 2012;26(11):4743-54.

60. Tamashiro K et al. Prenatal stress or high-fat diet increases susceptibility to diet-induced obesity in rat offspring. Diabetes. 2009;58(5):1116-25. This paper was especially useful in its consideration of stress-diet exposures, which are central to the current developmental origins of health and disease hypotheses.

61. Cooper R. Race in biological and biomedical research. Cold Spring Harbor Perspectives in Medicine. 2013. 3(11).

62. Kaufman J, Rushani D, Cooper R. Nature versus nurture in the explanations for racial/ethnic health disparities: parsing disparities in the era of genome-wide association studies, in reconsidering race: global and comparative studies in race and genomics, $\mathrm{K}$. Suzuki and D. von Vacano, Editors. 2015, Oxford University Press: London.

63. Soobader M et al. Levels of analysis for the study of environmental health disparities. Environ Res. 2006;102(2):172-80.

64. Norton $\mathrm{J}$ et al. Race, wealth, and solid waste facilities in North Carolina. Environ Health Perspect. 2007;115(9):1344-50. 
65. Jones MR et al. Race/ethnicity, residential segregation, and exposure to ambient air pollution: the Multi-Ethnic Study of Atherosclerosis (MESA). Am J Public Health. 2014;104(11): 2130-7.

66. Kariisa $\mathrm{M}$ et al. Differential ambient air pollution exposure in a chronic obstructive pulmonary disease cohort: the role of area-level socioeconomic factors. Environ Justice. 2014;7(1):18-26.

67. Laraia B et al. Neighborhood factors associated with physical activity and adequacy of weight gain during pregnancy. J Urban Health. 2007;84(6):793-806.

68. Laraia BA, et al. Direct observation of neighbourhood attributes in an urban area of the U.S. south. International Journal of Health Geographics. 2006. 5(11): p. online.

69. Miranda M, Messer L, Kroger G. The impact of neighborhood quality on pregnancy outcomes. Environ Health Perspect. 2011;12(3):471-7.

70. Sampson R, Raudenbush S. Seeing disorder: neighborhood stigma and the social construction of "broken windows". Soc Psychol Q. 2004;67(4):319-42.

71. LaVeist T, Wallace JJ. Health risk and inequitable distribution of liquor stores in African American neighborhood. Soc Sci Med. 2000;54(4):613-7.

72. Romley $\mathrm{J}$ et al. Alcohol and environmental justice: the density of liquor stores and bars in urban neighborhoods in the United States. J Stud Alcohol Drugs. 2007;68(1):48-55.

73. Powell L, Chaloupka F, Bao Y. The availability of fast-food and full-service restaurants in the United States: associations with neighborhood characteristics. Am J Prev Med. 2007;33(4 Suppl): S240-5.

74. Baker $\mathrm{E}$ et al. The role of race and poverty in access to foods that enable individuals to adhere to dietary guidelines. Prev Chronic Dis. 2006;3(3):A76.

75. Larson N, Story M, Nelson M. Neighborhood environments: disparities in access to healthy foods in the US. Am J Prev Med. 2009;36(1):74-81.

76. Richardson A et al. Are neighbourhood food resources distributed inequitably by income and race in the USA? Br Med J Open. 2012;2(2):e000698.

77. Powell L et al. Availability of physical activity-related facilities and neighborhood demographic and socioeconomic characteristics: a national study. Am J Public Health. 2006;96(9):1676-80.

78. Wolch J, Wilson J, Fehrenbach J. Parks and park funding in Los Angeles: an equity-mapping analysis. Urban Geogr. 2005;26(1):4 35 .

79. Moore $\mathrm{L}$ et al. Availability of recreational resources in minority and low socioeconomic status areas. Am J Prev Med. 2008;34(1):16-22.

80. Dubowitz T et al. Neighborhood socioeconomic status and fruit and vegetable intake among whites, blacks, and Mexican Americans in the United States. Am J Prev Med. 2008;87(6):1883-91.
81. Ford P, Dzewaltowski D. Disparities in obesity prevalence due to variation in the retail food environment: three testable hypotheses. Nutr Rev. 2008;66(4):216-28.

82. Lovasi $\mathrm{G}$ et al. Built environments and obesity in disadvantaged populations. Epidemiol Rev. 2009;31:7-20.

83. Gouda M. The long-term effect of slavery on violent crime: evidence from U.S. counties. Available at SSRN 2358389. 2013.

84. Joe J. Out of harmony: health problems and young native American men. J Am Coll Heal. 2001;49(5):237-42.

85. Gee G. A multilevel analysis of the relationship between institutional and individual racial discrimination and health status. Am J Public Health. 2002;92(4):615-23.

86. Pasco E, LS R. Perceived discrimination and health: a meta-analytic review. Psychological Bulletin. 2009. 135 (4).

87. Williams D, Mohammed S. Discrimination and racial disparities in health: evidence and research needed. J Behav Med. 2009;32(1): 20-47.

88. Harper C, Marcus R, Moore K. Enduring poverty and the conditions of childhood: life course and intergenerational poverty transmissions. World Dev. 2003;31(3):535-54.

89. Moore K. Thinking about youth poverty through the lenses of chronic poverty, life-course poverty and intergenerational poverty. 2005: Chronic Poverty Research Centre (CPRC)

90. Draxten $\mathrm{M}$ et al. Parental role modeling of fruits and vegetables at meals and snacks is associated with children's adequate consumption. Appetite. 2014;78:1-7.

91. Appleton A et al. Patterning in placental 11-B hydroxysteroid dehydrogenase methylation according to prenatal socioeconomic adversity. PLoS ONE. 2014;8(9):e74691.

92. Tehranifar $\mathrm{P}$ et al. Early life socioeconomic factors and genomic DNA methylation in mid-life. Epigenetics. 2013;8(1):23-7.

93. Brøns $\mathrm{C}$ et al. Effects of high-fat overfeeding on mitochondrial function, glucose and fat metabolism, and adipokine levels in lowbirth-weight subjects. Am J Physiol Endocrinol Metab. 2012;302(1):E43-51.

94. VanderWeele T. A unification of mediation and interaction-a 4 way decomposition. Epidemiology. 2014;25(5):749-61.

95. Kline R. Principles and practice of structural equation modeling. 2nd ed. New York: The Guilford Press; 2005.

96. Hammond R. Complex systems modeling for obesity research. Prev Chronic Dis. 2009;6(3):A97.

97. Rose G. Sick individuals and sick populations. Int J Epidemiol. 1985;14(1):32-8.

98. Swinburn B et al. The global obesity pandemic: shaped by global drivers and local environments. Lancet. 2011;378:804-14.

99. Guilarte $\mathrm{T}$ et al. Environmental enrichment reverses cognitive and molecular deficits induced by developmental lead exposure. Ann Neurol. 2003;53(1):50-6. 